\title{
Hemodynamic Modeling of the Intrarenal Circulation
}

\author{
K. M'rabet Bensalah, ${ }^{1}$ D. Uehlinger, ${ }^{2}$ R. Kalicki, ${ }^{2}$ and J. Czerwinska ${ }^{1}$ \\ ${ }^{1}$ ARTORG Center for Biomedical Engineering Research, University of Bern, Murtenstrasse 50, 3010 Bern, Switzerland; and \\ ${ }^{2}$ Department of Nephrology and Hypertension, University Hospital of Bern, Freiburgstrasse 15, 3010 Bern, Switzerland
}

(Received 17 May 2013; accepted 6 July 2013; published online 26 July 2013)

Associate Editor Nathalie Virag oversaw the review of this article.

\begin{abstract}
Three dimensional, time dependent numerical simulations of healthy and pathological conditions in a model kidney were performed. Blood flow in a kidney is not commonly investigated by computational approach, in contrast for example, to the flow in a heart. The flow in a kidney is characterized by relatively small Reynolds number $(100<R e<0.01$-laminar regime). The presented results give insight into the structure of such flow, which is hard to measure in vivo. The simulations have suggested that venous thrombosis is more likely than arterial thrombosis - higher shear rate observed. The obtained maximum velocity, as a result of the simulations, agrees with the observed in vivo measurements. The time dependent simulations show separation regimes present in the vicinity of the maximum pressure value. The pathological constriction introduced to the arterial geometry leads to the changes in separation structures. The constriction of a single vessel affects flow in the whole kidney. Pathology results in different flow rate values in healthy and affected branches, as well as, different pulsate cycle characteristic for the whole system.
\end{abstract}

Keywords-Kidney modeling, Hemodynamics, Renal pathology.

\section{INTRODUCTION}

While in vivo and in vitro experiments are well known and recognized methods in medical research, computational studies are only starting to get acceptance among physicians. This new method, known as in silico research, is rapidly gaining interest in the biomedical field due to its potential for speeding up the rate of discovery while reducing the need for expensive laboratory work and animal experiments. ${ }^{35}$

Renal disease is an important cause of mortality and morbidity worldwide with rapidly growing prevalence

Address correspondence to J. Czerwinska, ARTORG Center for Biomedical Engineering Research, University of Bern, Murtenstrasse 50, 3010 Bern, Switzerland. Electronic mail: justyna.czerwinska@ artorg.unibe.ch in emerging and Third World countries due principally to the increase of life expectancy and prevalence of diabetes and hypertension. Chronic kidney disease (CKD) is observed in about $25 \%$ of the population older than 64 years ${ }^{49}$ with almost $2 / 3$ of these people suffering primary from diabetic nephropathy, nephroangiosclerosis and arteriosclerotic lesions of larger vessels with secondary chronic ischemic kidney disease. ${ }^{41}$ All these pathological conditions involve primary the renal arterial vascular system ranging from the main supplying medium-sized renal artery to the glomerular and peritubular capillaries. Thus, diabetes is known to be the major risk factor for the development of macro- and microangiopathy and is responsible for the formation of stenotic lesions of the renal artery and as well as nodular diabetic glomerulosclerosis and intercapillary glomerulonephritis both affecting capillaries and adjacent structures. ${ }^{14}$ Arteriolosclerosis and hyalinosis, a lumen obliterating condition in renal arterioles, with secondary glomerular ischemia, are characteristic lesions in uncontrolled arterial hypertension. ${ }^{38}$ To a lesser extent, middle-sized intrarenal arteries and the venous system may also be affected by vasculitis and thromboembolism.9,19 The specific pathophysiological mechanisms and vascular structure involved in the aforementioned diseases, as well as the time course in the progression these conditions make the modeling of the renal circulation very appealing.

In this paper we have focuses on numerical investigations of the blood flow in the kidney model.

The pressure and flow distribution in the intrarenal circulation is of great interest given by the expected changes of the intrarenal vascular circulation in CKD. ${ }^{1}$

Vein and arteries follow Murray law ${ }^{23}$ concerning mass flow rate and branch order in the vascular tree. This observation was confirmed also by studying various animals and humans vascular system. ${ }^{22}$ 
The renal vasculature is a portal system: it is characterized by two capillary networks in series, namely, the glomerular and the peritubular. The blood enters from the renal artery with a pressure comparable to the systemic pressure in the abdominal aorta. The renal artery divides into segmental arteries feeding interlobar arteries. The latter divide into arcuate arteries characterized by being parallel to the kidney surface, and giving rise to numerous perforating radial arteries, which are the origin of the afferent and efferent arterioles respectively, feeding and draining the glomerular capillary bundle.

The model is based on the dimensional data of the rat kidney vasculature. The three-dimensional geometry of the renal arterial and venous tree is in accordance with the radii and lengths published by Nordsletten et al. ${ }^{25}$

The Strahler's order is used to describe the different segments of the renal vascular tree. It is one of the numerous ordering systems proposed to measure numerically the branching complexity of the vascular arborescence. It starts by labeling all terminal arterioles as order 0 , and then counts up following the vessel segments upstream.

According to Nordsletten et al., ${ }^{25}$ there is a tight correlation between this order and the vessel radius. Since blood flow through the systemic circulation is influenced most significantly by vessel diameter, this ordering provides insight into blood distribution.

The arterial and venous trees used for the simulation represent the macroscopic parts of the uni-lobar rat kidney. For the arterial tree it starts with one feeding renal artery, that trifurcates into three arteries (segmental or early interlobar), which then give rise to the long interlobar arteries. These bifurcate into different generations of arcuate arteries, which are parallel to the renal cortex. The same order has been used for the venous tree. Table 1 gives the Strahler's order of each segment.

According to Trueta et al. ${ }^{36}$ there is no fundamental difference between the minute vascular pattern of the individual lobules of the adult human kidney and the single lobule of the rabbit's kidney. Ufendahl et al. ${ }^{39}$ extended this statement to all mammal kidneys. Since rabbit and rat kidney are both unilobar,

TABLE 1. The Strahler's order given to the different arterial and venous segments.

\begin{tabular}{lll}
\hline Arterial segments & Strahler's order & Venous segments \\
\hline Feeding renal artery & 10 & Main vein \\
Interlobar arteries & $8 ; 9$ & Interlobar veins \\
Arcuate arteries & $7 ; 6$ & Arcuate veins \\
Interlobular arteries & $5 ; 4 ; 3 ; 2$ & Interlobular veins \\
Afferent arterioles & $1 ; 0$ & Venules \\
\hline
\end{tabular}

the vascular data of the rat kidney were used in this computational study to describe one lobule of the human kidney.

Some adaptation procedures were necessary. The feeding renal artery and the main renal vein of Table 1 are comparable to the lobular branches of the segmental arteries and veins. Therefore, the physical settings were calculated in order to create a vascular condition similar to the one found in a human renal lobule (cf. physical settings/pressure adaptation).

The aim of the present study was to create a hemodynamic model of the renal vascular system using the COMSOL Multiphysics Software. ${ }^{7}$ In order to define the vascular dimensions and flow characteristics of the kidney, data were first collected from literature. Based on the latter, the complex geometry of the intrarenal vessels was constructed. Afterwards, hemodynamic simulations in stationary and time-dependent conditions were performed, and the results analyzed. This permitted us to compare the in silico results to the in vivo and in vitro data found in literature, and thus, evaluate their significance.

Furthermore, pathological conditions were simulated by introducing modifications to the geometry such as a progressive arterial constriction as found in atherosclerosis.

\section{MATERIALS AND METHODS}

\section{Geometrical Model}

The geometry was constructed based on the average data given in literature. This idealized kidney model has allowed to study basic flow behavior. One of the most important steps in the modeling process is the creation of a good mesh, since the number of mesh elements determines the quality of the results as well as the computation time. It is essential to create an intelligent distribution of the mesh quality, making a finer mesh onto the areas of interest such as the inlet and outlet boundaries, and the bi- and trifurcation regions, while the mesh can be coarser on the remaining regions. The number of elements used for computation was above $2,300,000$. The simulations were performed on a 32 core computer and has required $24 \mathrm{~GB}$ of memory. The computational time for each case was a bit more than $24 \mathrm{~h}$ (between 87,000 and $88,000 \mathrm{~s}$ ).

The fluid was defined as an incompressible Newtonian liquid with a density of $1059.5 \mathrm{~kg} \mathrm{~m}^{-3}$ and a dynamic viscosity of $0.003 \mathrm{~Pa} \mathrm{~s}$. These values are comparable to the ones found in human blood. ${ }^{21}$

Two types of studies were used, first stationary and then time dependent. 


\section{Stationary Study}

Boundary conditions were determined as a constant inlet pressure of $p_{\text {in }}=12,621 \mathrm{~Pa}$, and an outlet pressure of $p_{\text {out }}=12,491 \mathrm{~Pa}$ in the arterial tree. The inlet value was adapted from the mean pressure in the human renal artery, equivalent to $12,799 \mathrm{~Pa}$ $(96 \mathrm{mmHg})$ according to the literature. ${ }^{44}$ The outlet pressure was deliberately defined according to Ufendahl et al.'s ${ }^{39}$ statement that the pressure drop up to the arcuate arteries was negligible. The pressure drop was assumed to be about $1.6 \%$. For the venous tree the inlet pressure $p_{\text {in }}$ was set at $740 \mathrm{~Pa}$ and the outlet pressure, $p_{\text {out }}$, at $547 \mathrm{~Pa}$.

\section{Time Dependent Study}

The time dependent study was restricted to the arterial tree. We determined the inlet boundary condition as a variable pressure with the following time dependent function:

$$
p(t)=\left[\left(p_{\text {sys }}-p_{\text {dias }}\right) 0.5 f(t)\right]+p_{\text {dias }}
$$

where $p_{\text {sys }}$ was set at $14,000 \mathrm{~Pa}(105 \mathrm{mmHg})$ and $p_{\text {dias }}$ at $10,488 \mathrm{~Pa}(78.6 \mathrm{mmHg})$, corresponding to the pressures at the entry of the lobule for systemic systolic and diastolic pressures of $14,265 \mathrm{~Pa}(107 \mathrm{mmHg})$ and $10,666 \mathrm{~Pa} \quad(80 \mathrm{mmHg})$ respectively (cf. "Pressure Adaptation" section), and $f(t)$ is a periodic function of a pulsatile cycle as described in Vasava et al.'s study of the human pulse. ${ }^{42}$ The frequency was set at 60 beats/ min $(1 \mathrm{~Hz})$. Table 2 describes function $f(t)$.

We have modified this function to make it continuous for every time step. The outlet pressure $p_{\text {out }}$ remains constant at 10,320 Pa.

For the detailed studies of the time dependent flow, specific points and branches were chosen. The definition of that geometrical setup can be found in Fig. 1.

\section{Pressure Adaptation}

Considering the geometry equivalent to one lobule of the human kidney, we had to adapt the flow characteristics of the model.

According to Poiseuille's law:

$$
\Delta P=R Q
$$

TABLE 2. The equations and variables of the piecewise function $f(t)$ used in the time-dependent study.

\begin{tabular}{ll}
\hline Time period $[\mathrm{s}]$ & \multicolumn{1}{c}{ Function } \\
\hline $0-0.3$ & $6.05 t^{2}$ \\
$0.3-0.75$ & $0.75-0.25 \cos (4 \pi(t-0.25))$ \\
$0.75-0.9$ & $-0.5 \sin (2 \pi t)$ \\
$0.9-1$ & $30\left(1-t^{2}\right)$ \\
\hline
\end{tabular}

$R$ is the resistance of the system:

$$
R=8 \mu L / \pi r^{4},
$$

where $\mu$ is the dynamic viscosity; $L$ is the length of the pipe. According to the literature the length of the human renal artery, $L_{\mathrm{RA}}$, is about $0.04 \mathrm{~m} ; Q$ is the blood flow. According to the literature the renal blood flow in humans, $Q_{\mathrm{RA}}$, is about $1 \mathrm{~L} \mathrm{~min}^{-1}$ $\left(0.0000167 \mathrm{~m}^{3} \mathrm{~s}^{-1}\right) ; r$ is the radius of the pipe. According to the literature the diameter of the human renal artery is about $5 \mathrm{~mm}$ (cf. Tables 3,4 ). Though, the radius $r_{\mathrm{RA}}$ is $0.0025 \mathrm{~m}$.

Using these values, the resistance in the renal artery is: $R_{\mathrm{RA}}=7,822,784 \mathrm{~Pa} \mathrm{~s} \mathrm{~m}^{-3}$.

The renal artery divides into five segmental arteries before entering the lobules. These arteries are in parallel. Assuming that there is the same resistance in each segmental artery (SA), the main resistance of the segmental part (TSA) will be: $R_{\mathrm{TSA}}=R_{\mathrm{SA}} / 5$.

The length $L_{\mathrm{SA}}$ is $0.03 \mathrm{~m}^{30}$ and the radius $r_{\mathrm{SA}}$ is $0.002 \mathrm{~m}$, therefore we find $R_{\mathrm{SA}}=14,323,945 \mathrm{~Pa} \mathrm{~s} \mathrm{~m}^{-3}$ and in consequence $R_{\mathrm{TSA}}=2,864,789 \mathrm{~Pa} \mathrm{~s} \mathrm{~m}^{-3}$, though, $\Delta P=\left(R_{\mathrm{RA}}+R_{\mathrm{TSA}}\right) Q=178 \mathrm{~Pa}$. So the pressure difference drops by $1.4 \%$ between the entry of the kidney and the artery at which the geometry of the model begins.

The equations were solved with a GMRES solver with preconditioning. ${ }^{7}$

\section{Simulation of Pathologic Conditions}

Based on the sub-mentioned time-dependent simulation, pathologic conditions were created by introducing a constriction region to one of the three main arteries (Strahler's order 9). The introduced obstruction was the result of the intersection between a newly added sphere and the arterial tree model. The intersection volume was increased gradually to three constriction levels as shown in exact value in Table 5.

Using the physical settings and features described above, a model of the renal vascular system representing the perfusion of one lobule up to the arcuate arteries was created.

\section{RESULTS}

The largest resistance to flow in the renal arterial tree is encountered within the interlobular arteries and the arterioles as described in Table 5. The pressure values of the different vascular segments of Table 6 are independent of each other. This explains the non-linear distribution of pressure. Nevertheless, the literature data are unified in giving the largest pressure loss to the interlobular region, also called, the radial arteries, due 


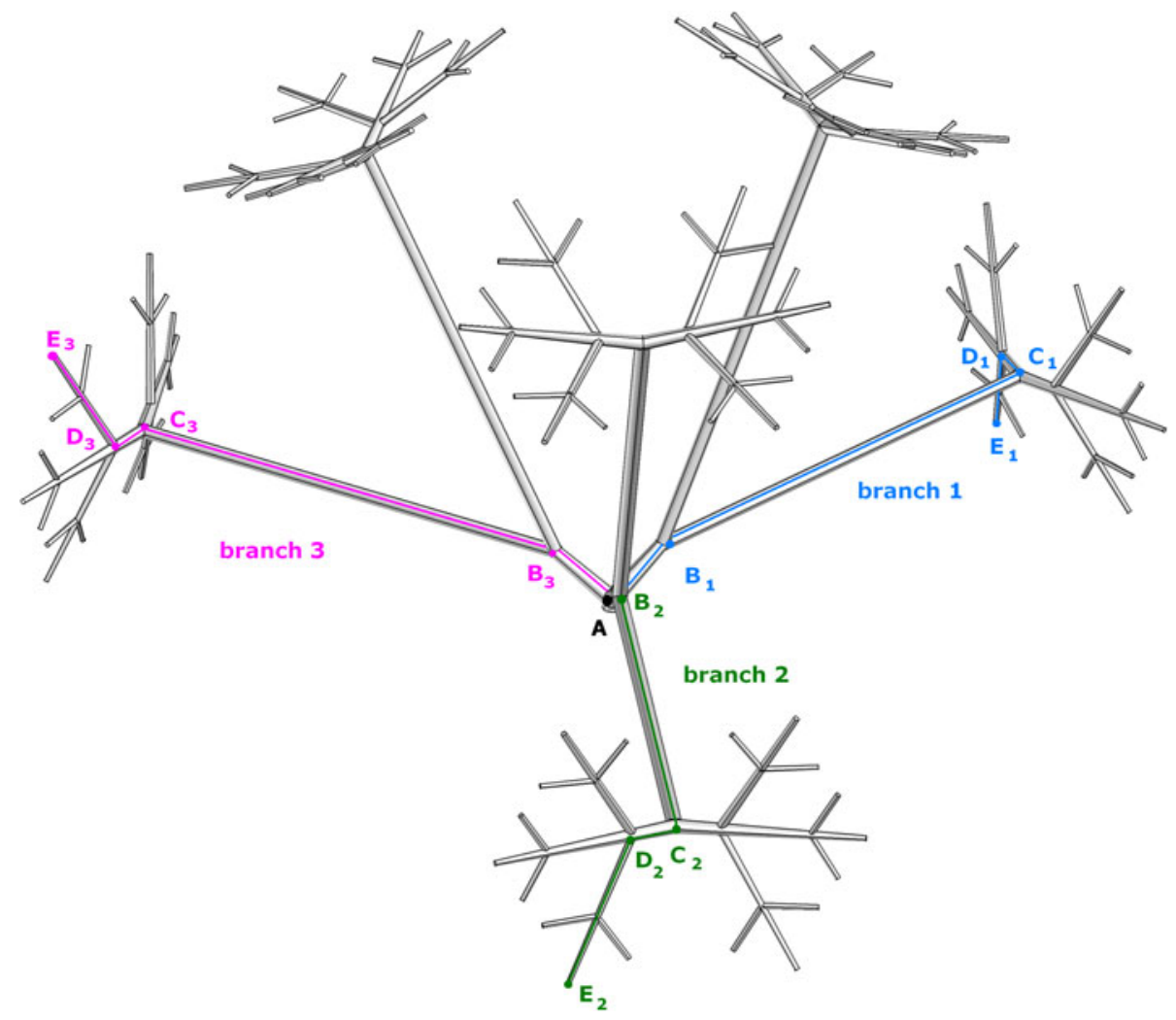

FIGURE 1. Arterial tree measurement point selection.

to their perpendicular and perforating position to the renal surface. This configuration, added to their highly muscular wall, makes them the first resistance site of interest in the renal circulation. ${ }^{39}$

According to Pries et al., ${ }^{28}$ the contribution of microvessels to total peripheral resistance is strongly affected by diameter-dependent variation of blood viscosity. This phenomenon is known as the FahraeusLindqvist effect. It says that the decrease of apparent blood viscosity continues down to a diameter of $10 \mu \mathrm{m}$. After that, a steep increase of viscosity is observed for diameters approaching the minimum cylindrical diameter of normal human erythrocytes. The hematocrit drops down in capillaries to $10-12 \%$ and this additionally influences viscosity. We have focused on the vascular system of the much larger scale than a few RBC diameters. Hence, the viscosity was chosen as constant.

Renal arterial flow has been measured extensively. Despite the different geometries the velocity profile in different aortorenal bifurcation was very similar. ${ }^{46}$ Hence, we have chosen only one standard profile. The maximal velocity was measured in dog feeding arteries $(0.6 \mathrm{~m} / \mathrm{s}$ with the pulse cycle tree time faster than for humans) ${ }^{15}$ For humans the maximal observed velocity was $0.75 \mathrm{~m} / \mathrm{s}^{3}$ and $0.6-0.7 \mathrm{~m} / \mathrm{s},{ }^{45}$ and $0.52 \mathrm{~m} / \mathrm{s} .{ }^{47}$ This confirms our assumption with respect to the choice of the pressure drop and resulting flow velocity.

The simulations of physiological and pathological conditions were performed. Physiological conditions included stationary and time dependent flow. Pathology was introduced as a constriction of the arterial vessel. Thrombosis in arteries and veins can be observed from damages to the vessels, slowing of the blood flow (stasis) and an increased coagulability of the blood. In the kidney vein thrombosis were reported in. ${ }^{2,29}$ Renal arterial stent induced thrombosis was described in Dobbeleir et al. ${ }^{11}$ and Cai et al. ${ }^{5}$

The physiological condition results show parameters at which the kidney can operate. Especially important for understanding flow conditions in the kidney are pressure and shear rate. Adequate pressure results in a sufficient flow and shear rate indicates area, which are more prone for obstruction formation and flow separation regions. Figure 2 shows the arterial tree pressure and shear rate distribution. The surface shear rate is maximal at the bi- and trifurcation areas. The largest pressure drops are along the longest branches. The highest shear rate is present in junctions and it is relatively low. Thrombosis generally occurs if the shear rate is of the order of magnitude larger than the one observed in our paper. ${ }^{24}$ 
TABLE 3. Collected data from literature review for the renal vascular system in human.

\begin{tabular}{|c|c|c|c|c|c|}
\hline & Ref & Number per kidney & Diameter (mm) & Length (mm) & Pressure $(\mathrm{mmHg})$ \\
\hline \multirow[t]{3}{*}{ Abdominal aorta } & $x$ & 1 & $>10$ & & \\
\hline & 44 & & $21.6 \pm 1.6(18-24)$ & & \\
\hline & 37 & & Men 18.33, Women 15.89 & & \\
\hline \multirow[t]{9}{*}{ Renal artery } & $\mathrm{x}$ & 1 & $0.3-10$ & & \\
\hline & 39 & & & & 120 \\
\hline & 43 & & $7.9(4-12)$ & & \\
\hline & 16 & & $5-6$ & $40-60$ & \\
\hline & 33 & & Left: $6.0 \pm 0.5$, & Left: $39.9 \pm 6.7$ & \\
\hline & 32 & & $\begin{array}{l}\text { Right: } 5.6 \pm 0.6 \\
4.87\end{array}$ & $\begin{array}{l}\text { Right: } 44.9 \pm 7.4 \\
\text { feft. } 286 . \text { Biaht } 346\end{array}$ & \\
\hline & 30 & & 7 & $\begin{array}{l}\text { Lett: 28.6; Rignt } 34.6 \\
28\end{array}$ & \\
\hline & 37 & & $\begin{array}{l}\text { Men: Right: } 5.09 \text {, Left: } 5.14 \text {, } \\
\text { Women: Right: } 4.59 \text {, Left: } 4.66\end{array}$ & & \\
\hline & 44 & & $5.9 \pm 1.3(3.0-7.0)$ & & $96 \pm 10(79-113)$ \\
\hline Anterior branch & $\mathrm{x}$ & 1 & $0.3-10$ & & \\
\hline Posterior branch & $\mathrm{x}$ & 1 & $0.3-10$ & & \\
\hline \multirow{2}{*}{$\begin{array}{l}\text { Segmental artery (apex, } \\
\text { upper ant, middle ant, } \\
\text { lower pole) }\end{array}$} & $\mathrm{x}$ & 5 & $0.3-10$ & & \\
\hline & 30 & & 6 & $29-33$ & \\
\hline Interlobular artery & $\mathrm{x}$ & & $0.3-10$ & & \\
\hline Arcuate artery & $\mathrm{x}$ & & $0.3-10$ & & \\
\hline \multirow[t]{2}{*}{ Interlobular artery/radial arteries } & $x$ & & $10-300 \times 10^{-3}$ & & \\
\hline & 39 & & $20-50 \times 10^{-3}$ & & 120 drops to 80 \\
\hline \multirow[t]{2}{*}{ Afferent arteriole } & $\begin{array}{l}x \\
39\end{array}$ & $10^{6}$ & $10-300 \times 10^{-3}$ & & 60 \\
\hline & 13 & & $\begin{array}{l}\text { Cortical } 15 \times 10^{-3} \\
\text { JM } 2 \times 10^{-2}\end{array}$ & & \\
\hline \multirow[t]{2}{*}{ Glomerulus } & $\mathrm{x}$ & $10^{6}$ & & & \\
\hline & 39 & & $280-287 \times 10^{-3}$ & & $50-60$ \\
\hline \multirow[t]{4}{*}{ Efferent arerioles } & $x$ & & $10-300 \times 10^{-3}$ & & 60 drops to 15 \\
\hline & 39 & $10^{6}$ & $16-33 \times 10^{-3}$ & & \\
\hline & 13 & & Cortical $1 \times 10^{-2}$ & & \\
\hline & & & $\mathrm{JM} 20-25 \times 10^{-3}$ & & \\
\hline \multirow[t]{3}{*}{ Pertibular capillary bed } & $\mathrm{x}$ & & $5-10 \times 10^{-3}$ & & $10-15$ \\
\hline & 39 & & & & \\
\hline & 13 & & $7 \times 10^{-3}$ & & \\
\hline Stellar vein & $x$ & & $50-200 \times 10^{-3}$ & & \\
\hline Vasa recta ascendens & $x$ & & $5-10 \times 10^{-3}$ & & \\
\hline \multirow[t]{2}{*}{ Vasa recta descendens } & $x$ & & $5-10 \times 10^{-3}$ & & \\
\hline & 13 & & $12-15 \times 10^{-3}$ & & \\
\hline \multirow[t]{2}{*}{ Interlobular vein } & $x$ & & $50-200 \times 10^{-3}$ & & \\
\hline & 39 & & & & $5-10$ \\
\hline Arcuate vein & $x$ & & $50-200 \times 10^{-3}$ & & \\
\hline Interlobar vein & $x$ & & $0.2-10$ & & \\
\hline Renal vein & $x$ & 1 & $0.2-10$ & & \\
\hline Vena cava & $\mathrm{x}$ & 1 & $>10$ & & \\
\hline
\end{tabular}

Reference $\mathrm{x}$ and italic writing stand for generally accepted values taken from medical lectures and textbooks. JM: Juxtamedullary.

Figure 2 shows pressure and shear rate results for the venous tree. The largest pressure drop occurs in the small vessels. The pressure is much smaller, although the pressure drop is comparable to the arterial tree, and it results in a much larger shear rate. Again, the junctions are the regions with increased shear rate. As expected from clinical observation, the venous tree is more prone to thrombosis formation than the arterial tree. Nevertheless, physiological conditions are far away from the ones observed during thrombosis formation.

At maximal pressure difference between the inlet and outlet (time $0.5 \mathrm{~s}$ ) a secondary flow (vortices) was observed in the proximal portion of the arterial branches. Such a secondary flows may result from transverse pressure gradients. ${ }^{24}$ This phenomenon might be the result of a physiologic flow reversal, as it is seen in some pulsatile flow studies. ${ }^{31}$ 
TABLE 4. Collected data from literature review about the renal vascular system in the rat.

\begin{tabular}{|c|c|c|c|c|c|c|c|}
\hline & Ref. & $\begin{array}{l}\text { Number } \\
\text { per kidney }\end{array}$ & $\begin{array}{l}\text { Diameter } \\
(\mu \mathrm{m})\end{array}$ & $\begin{array}{l}\text { Length } \\
(\mathrm{mm})\end{array}$ & $\begin{array}{c}\text { Pressure } \\
(\mathrm{mmHg})\end{array}$ & $\begin{array}{c}\text { Pressure } \\
\text { drop }(\mathrm{mmHg})\end{array}$ & Viscosity \\
\hline Abdominal aorta & 26 & & & & $125 \pm 3.8$ & & \\
\hline \multirow[t]{4}{*}{ Renal artery } & 4 & 1 & & & 95 & & * \\
\hline & 25 & & 432.2 & 0.185 & & & 0.00298 \\
\hline & $12^{* *}$ & & & & 112.5 & & \\
\hline & $39^{\star *}$ & & & & 115 & -0.5 & \\
\hline \multirow[t]{2}{*}{ Segmental artery } & 25 & 3 & 382.84 & 1.44 & & & 0.00295 \\
\hline & $39^{* *}$ & & & & 115 & -0.1 & \\
\hline \multirow[t]{2}{*}{ Interbolar artery } & $39^{* *}$ & & & & 115 & -0.1 & \\
\hline & 25 & 6 & 279.66 & 8.975 & & & 0.00285 \\
\hline \multirow[t]{5}{*}{ Arcuate artery } & $12^{* *}$ & & & & 110 & & \\
\hline & 6 & & & & $95.6 \pm 1.4$ & & \\
\hline & 25 & $24-90$ & $107.74-172.3$ & $1.031-2.516$ & & & $0.00263-0.00238$ \\
\hline & 17 & & $438 \pm 26$ & & & & \\
\hline & $39^{\star \star}$ & & & & 115 & -2 & \\
\hline \multirow[t]{4}{*}{ Interlobular artery } & 25 & $247-4373$ & $40.12-88.46$ & $0.404-0.511$ & & & $0.00227-0.00182$ \\
\hline & 17 & & $64 \pm 4$ & & & & \\
\hline & $12^{\star \star}$ & & & & 110 & & \\
\hline & $39^{* *}$ & & & & $115-80$ & -35 & \\
\hline \multirow[t]{5}{*}{ Afferent arteriole } & 6 & & & $0.05-0.150$ & $91.6 \pm 4.6-79.5 \pm 4.1$ & & \\
\hline & 25 & $13,070-29,566$ & $20.16-27.8$ & $0.312-0.423$ & & & $0.00164-0.00149$ \\
\hline & 17 & & $20-30 \pm 1$ & & & & \\
\hline & $12^{\star \star}$ & & & & $92-68$ & & \\
\hline & $39^{\star *}$ & & & & $80-57$ & -32 & \\
\hline \multirow[t]{5}{*}{ Glomerulus } & 18 & & $4.7-6.5-9.9$ & & & & \\
\hline & 8 & & & & $45.5 \pm 3.6$ & & \\
\hline & 6 & & & & $48.5 \pm 2.9$ & & \\
\hline & $12^{* *}$ & & & & 49 & & \\
\hline & $39^{\star *}$ & & & & 57 & & \\
\hline \multirow[t]{5}{*}{ Efferent arerioles } & 4 & & & & $10-15$ & & \\
\hline & 12 & & & & & $30-40$ & \\
\hline & 6 & & & & $31.3 \pm 3.8-16.2 \pm 0.9$ & & \\
\hline & $12^{\star *}$ & & & & $32-15$ & & \\
\hline & $39^{\star *}$ & & & & $57-15$ & & \\
\hline \multirow[t]{4}{*}{ Pertibular capillary bed } & 6 & & & & $3.9 \pm 0.5$ & & \\
\hline & 12 & & & & $15-20$ & $30-40$ & \\
\hline & $12^{\star \star}$ & & & & 9 & & \\
\hline & $39^{* *}$ & & & & $15-12$ & & \\
\hline Stellar vein & 6 & & & & $4.1 \pm 1.4$ & & \\
\hline Vasa recta & 6 & & & & $16.2 \pm 0.9$ & & \\
\hline descendens & 50 & & $14.3 \pm 0.9$ & & & & \\
\hline Vasa recta & 6 & & & & $6.3 \pm 0.4$ & & \\
\hline ascendens & 50 & & $17.9 \pm 0.9$ & & & & \\
\hline \multirow[t]{2}{*}{ Venules } & ** & & & & & -3 & \\
\hline & 25 & $68,564-30,659$ & $21.58-29.44$ & $0.155-0.248$ & & & $0.00152-0.00166$ \\
\hline \multirow[t]{3}{*}{ Interlobular vein } & 6 & & & & $4.1 \pm 1.4$ & & \\
\hline & 25 & $9258-418$ & $52.32-138$ & $0.315-1.054$ & & & $0.00197-0.00252$ \\
\hline & $39^{* *}$ & & & & 7 & -2 & \\
\hline \multirow[t]{3}{*}{ Arcuate vein } & $\star \star$ & & & & & -1 & \\
\hline & 6 & & & & $4.1 \pm 1.4$ & & \\
\hline & 25 & $139-38$ & $228-354$ & $1.147-1.695$ & & & $0.00276-0.00292$ \\
\hline \multirow[t]{3}{*}{ Interlobular vein } & ** & & & & & -0.1 & \\
\hline & 6 & & & & $4.1 \pm 1.4$ & & \\
\hline & 25 & 9 & $570-856.1$ & $6.131-3.091$ & & & $0.00303-0.00307$ \\
\hline \multirow[t]{4}{*}{ Renal vein } & $\star \star$ & & & & & -1 & \\
\hline & 4 & & & & 5 & & * \\
\hline & 25 & 2 & 1207.54 & 3.12 & & & 0.00309 \\
\hline & $12^{\star *}$ & & & & 6 & & \\
\hline Vena cava & 26 & & & & $5.6 \pm 0.13$ & & \\
\hline
\end{tabular}

${ }^{*}$ Values deduced from plots. 
Figure 3 shows the time dependent study for the physiological and the most severe pathological condition. The pressure variation shows the dependence of the position in the arterial tree and follows a gradual proximal to distal distribution. The time-dependent variation of pressure is comparable to the data found in literature. The pressure drop behaves similarly as in the steady state simulations. The shear rate values are however much larger due to the fact that the time dependent variation in flow velocity introduces vortex formation. The pathological condition is visibly influencing branch where the constraints were introduced. To better understand the differences between these states more analysis were performed, showing point values of specific parameters.

Figure 3 shows the time distribution of the magnitude of the velocity at the points given by Fig. 1 . Branch 3 is the branch not affected by any pathology. Branch 1 is the one where the geometrical obstruction was introduced. The pathological condition results in

TABLE 5. Constriction dimensions area in the narrowest point.

\begin{tabular}{lcc}
\hline & $\begin{array}{c}\text { Intersection } \\
\text { area }\left(\mathrm{m}^{2}\right)\end{array}$ & $\begin{array}{c}\text { Area in \% from } \\
\text { normal condition }\end{array}$ \\
\hline Normal condition & $6.25 \times 10^{-8}$ & 100 \\
Pathology 1 & $5.83 \times 10^{-8}$ & 93 \\
Pathology 2 & $3.65 \times 10^{-8}$ & 58 \\
Pathology 3 & $0.35 \times 10^{-8}$ & 6 \\
\hline
\end{tabular}

an increase of pressure in branch one. This is especially well visible at point B1. Another important result is that an induced pathological condition has only a minimal effect on other branches (branch two is identical with branch three). Only a very restrictive pathological condition such as pathology number three significantly effects a pressure drop and only in the branch with the pathology. The boundary conditions of the flow ensure that the pressure drop is constant. The obstruction reduces the diameter of the vessels, hence the flow velocity must increases. Figure 4 shows the velocity changes and it can be seen that indeed the velocity magnitude after the obstruction is higher than in the healthy branch. In addition there is a higher flow through the healthy branch. The velocity is time dependent and correlated with the vortex structures present in pathological and healthy conditions. Hence the changes at point $\mathrm{B}$ are significant. This is better seen in the following pictures presenting vortex structures. For the normalized velocity magnitude a shift in the cycle can be noticed. The maximum velocity still is at the same place, but the flow after it differs especially for the condition with a significant obstruction. It can be seen for pathology number 3 , that both healthy and disturbed branches, are affected by the cycle shift, especially the healthy one, which needs to support an increased mass flow rate.

Reynolds numbers are in the range of values characteristic for laminar flow with separation for points A-C and creep flow for points D, E. Similar to the

TABLE 6. Average values of pressure distribution in the rat renal vascular tree taken from literature.

\begin{tabular}{|c|c|c|c|c|c|}
\hline & Strahler's order & Diameter $(\mu \mathrm{m})$ & Pressures $(\mathrm{Pa})$ & Standard deviation $(\mathrm{Pa})$ & Pressures $(\mathrm{mmHg})$ \\
\hline Renal artery & -10 & 4322 & 14,332 & 1000 & 107.5 \\
\hline \multirow[t]{2}{*}{ Interlobar artery } & -9 & 3828.4 & - & - & - \\
\hline & -8 & 2796.6 & - & - & - \\
\hline \multirow{2}{*}{ Arcuate artery } & -7 & 1723 & 14,247 & 1501 & 106.86 \\
\hline & -6 & 1077.4 & ** & $\star \star$ & ** \\
\hline \multirow[t]{4}{*}{ Interlobular artery } & -5 & 884.6 & 14,999 & 333 & 112.5 \\
\hline & -4 & 785.8 & * & * & * \\
\hline & -3 & 597.4 & * & * & * \\
\hline & -2 & 401.2 & 10,666 & - & 80 \\
\hline \multirow[t]{2}{*}{ Afferent arteriole } & -1 & 278 & 11,714 & 552 & 87.86 \\
\hline & & 201.6 & 9087 & 1512 & 68.16 \\
\hline Glomerulus & 0 & 6.05 & 6649 & 950 & 49.875 \\
\hline \multirow[t]{2}{*}{ Venules } & 0 & 294.4 & - & - & - \\
\hline & 1 & 523.2 & - & - & - \\
\hline \multirow[t]{4}{*}{ Interlobular veins } & 2 & 802.6 & 740 & 193 & 5.55 \\
\hline & 3 & 1006 & ** & ** & ** \\
\hline & 4 & 1380 & $* *$ & ** & ** \\
\hline & 5 & 2280 & ** & ** & ** \\
\hline \multirow[t]{2}{*}{ Arcuate veins } & 6 & 3540 & 547 & 187 & 4.1 \\
\hline & 7 & 5700 & ** & $* *$ & $* *$ \\
\hline \multirow[t]{2}{*}{ Interlobar veins } & 8 & 8561 & ** & ** & ** \\
\hline & 9 & $12,075.4$ & ** & $* *$ & ** \\
\hline Renal vein & 10 & $29,440.0$ & 733 & 67 & 5.5 \\
\hline
\end{tabular}

${ }^{*}$ Same value as on top; *intermediate value; - no data available. 

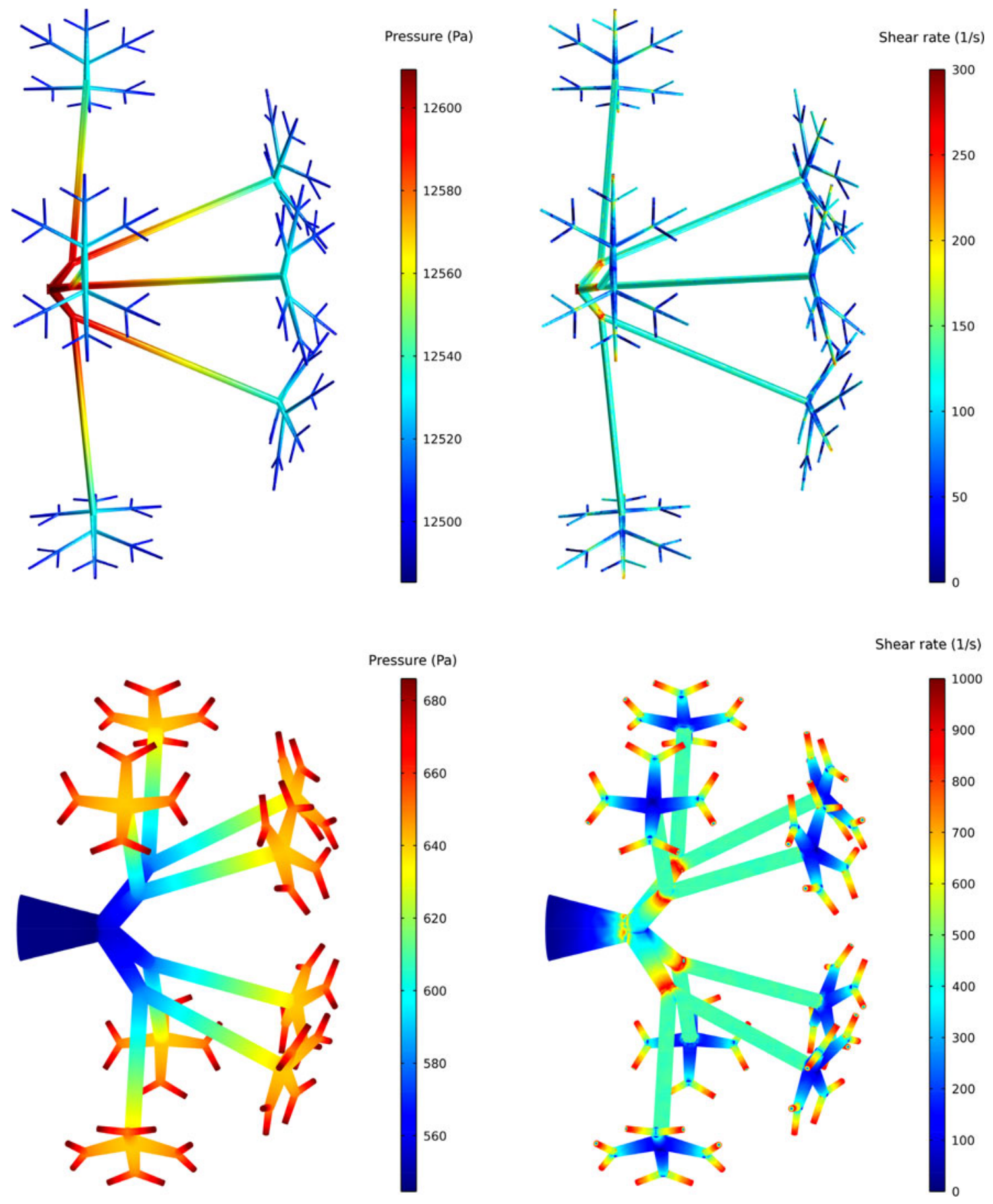

FIGURE 2. Pressure in ( $\mathrm{Pa})$ (left) and shear rate (1/s) (right) in renal arterial (top) and venous (bottom) tree.

results of the pressure distribution only a very severe pathology influences the Reynolds number distribution. The maximal inflow Reynolds number is between 70 and 90.

Figure 5 presents pressure, Reynolds numbers and the velocity magnitude along the length from the entrance at two times $(0.5$ and $0.8 \mathrm{~s}$ within the cycle). The characteristics are similar for both times and strongly depend on the induced constriction. The effect depends on the magnitude of the constriction and on the affected branch (branch 1)-flow differs significantly for pathology 2 and pathology 3 . In addition the healthy branch for the case of the most severe restriction (pathology 3 ) was plotted. Changes in velocity are visible and significant close to the constriction region. This is due to the different flow patterns induced by the pathology.

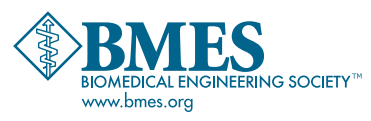




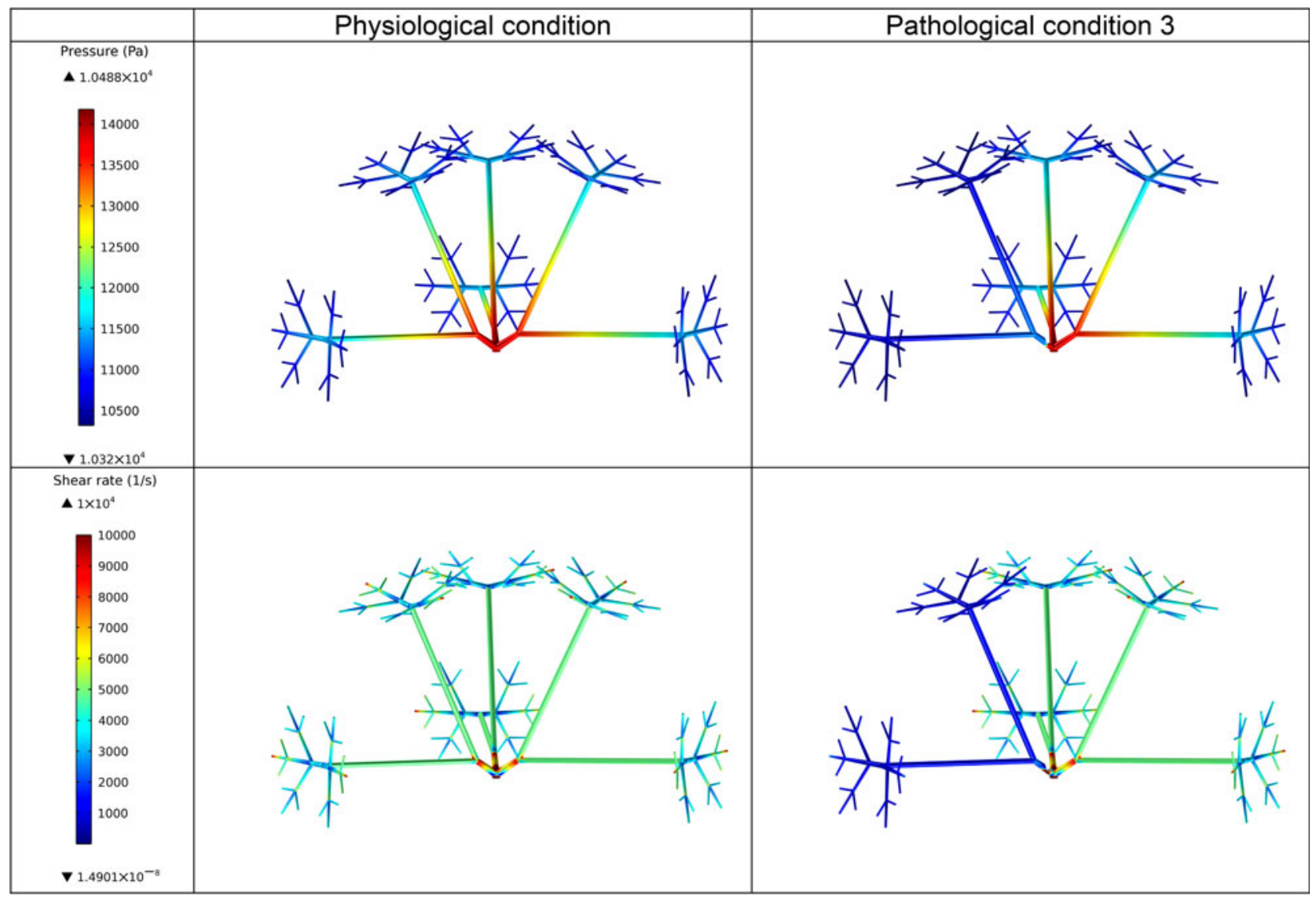

FIGURE 3. Pressure and shear rate for physiological condition and pathological condition 3 for the time of $0.5 \mathrm{~s}$.

Figures 6 and 7 show streamline distributions for various cuts to illustrate how the pathology influences the three-dimensional flow structures. Figure 6 presents time dependent frames showing the constriction influence. For the most severe one flow is being directed to a different branch. Figure 7 is a zoom picture of the similar view. It can be seen that at time $0.5 \mathrm{~s}$ symmetric vortices are observed. In pathological conditions this symmetry is gone, as expected. However, when the asymmetry in constrains raises, an asymmetric flow is induced and separation is not present even in the healthy branch. This means that the more severe pathology influences the global flow structures. It can be seen that other separation patterns are present during pathological conditions in the neighboring healthy branches.

The fact that this separation occurs only during systole but not during the rest of the cycle, speaks against a fully developed separation phenomenon according to Despard and Miller. ${ }^{10}$ A full separation would be observable during the entire pulsatile cycle.

Figure 8 shows mass flow rate curves as a function of time. First is the mass flow rate value in the main branch $\left(Q_{0}\right)$ in relations to the split values $\left(Q_{1}\right)$. At normal conditions the time curve follows the imposed impulse characteristic. The mass flow rate characteristics for the pathological conditions are also plotted in relation to the healthy and pathological branches. For normal conditions this would be a constant value. However, the observed difference is in the value as well as in the pulse characteristics. This is most visible for the pathology 3 , but changes in the pulse characteristic can already distinguish between pathology 1 and pathology 2.

\section{DISCUSSION}

A model representing part of the renal vascular system was created with the COMSOL multiphysics software. By defining inlet flow in a stationary and time-dependent manner, different aspects of the renal perfusion such as the pressure distribution in the arterial tree, the velocity magnitude at different sections, and the zones of high surface shear rate were studied. By calculating the Reynolds number, the flow regime was predicted laminar. Further simulations on pathologic conditions showed a pressure drop and velocity increase in the constricted region. 
Branch 1
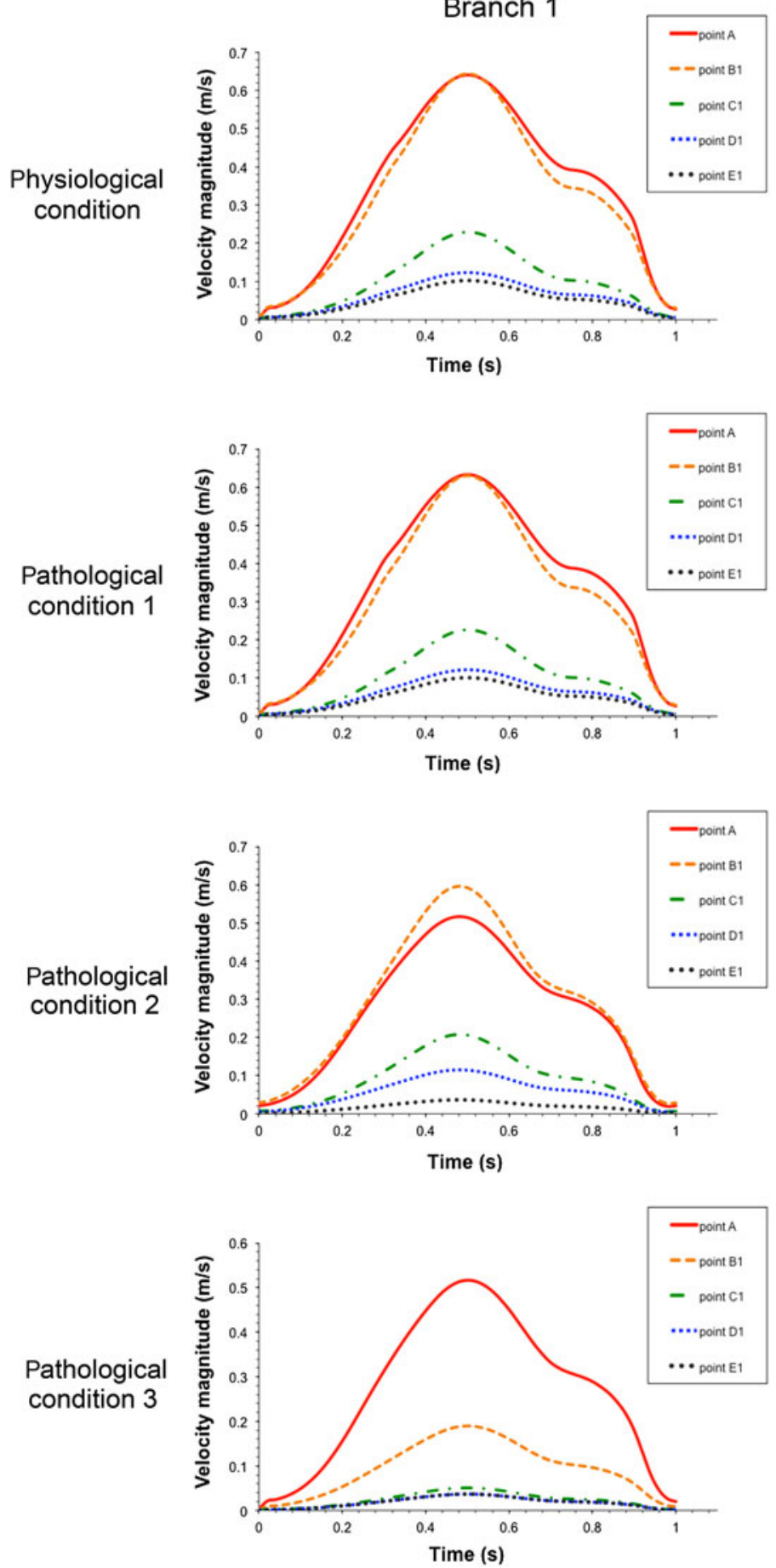
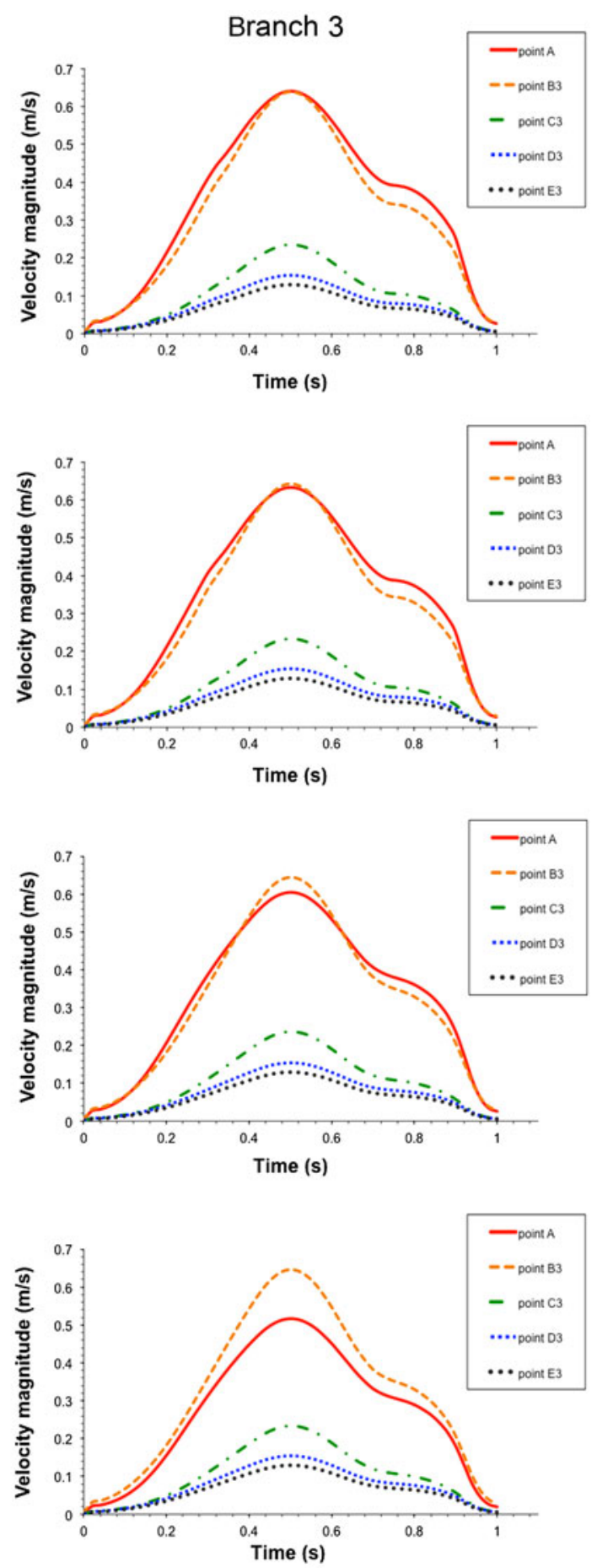

FIGURE 4. Velocity magnitude distribution in arterial tree in points defined in Fig. 1.

Renal artery stenosis was extensively investigated in recent years. Examples: CFD simulations to estimate pressure drop ${ }^{20,48}$ using turbulence models to predict precise vortex separation structures. In our model the obstruction is introduced further downstream in the kidney in the laminar flow regime. Additionally, we are following the influence of the obstruction on the whole kidney blood flow, which was not presented until now in modeling studies except in some animal experiments. For example, the kidney filtration in swine was reduced significantly by stenosis. ${ }^{40}$ In another experiment, electron beam computerized tomography was applied to look at flow in the obstructed kidney. ${ }^{27}$ However, the existing results give only a global view of the 

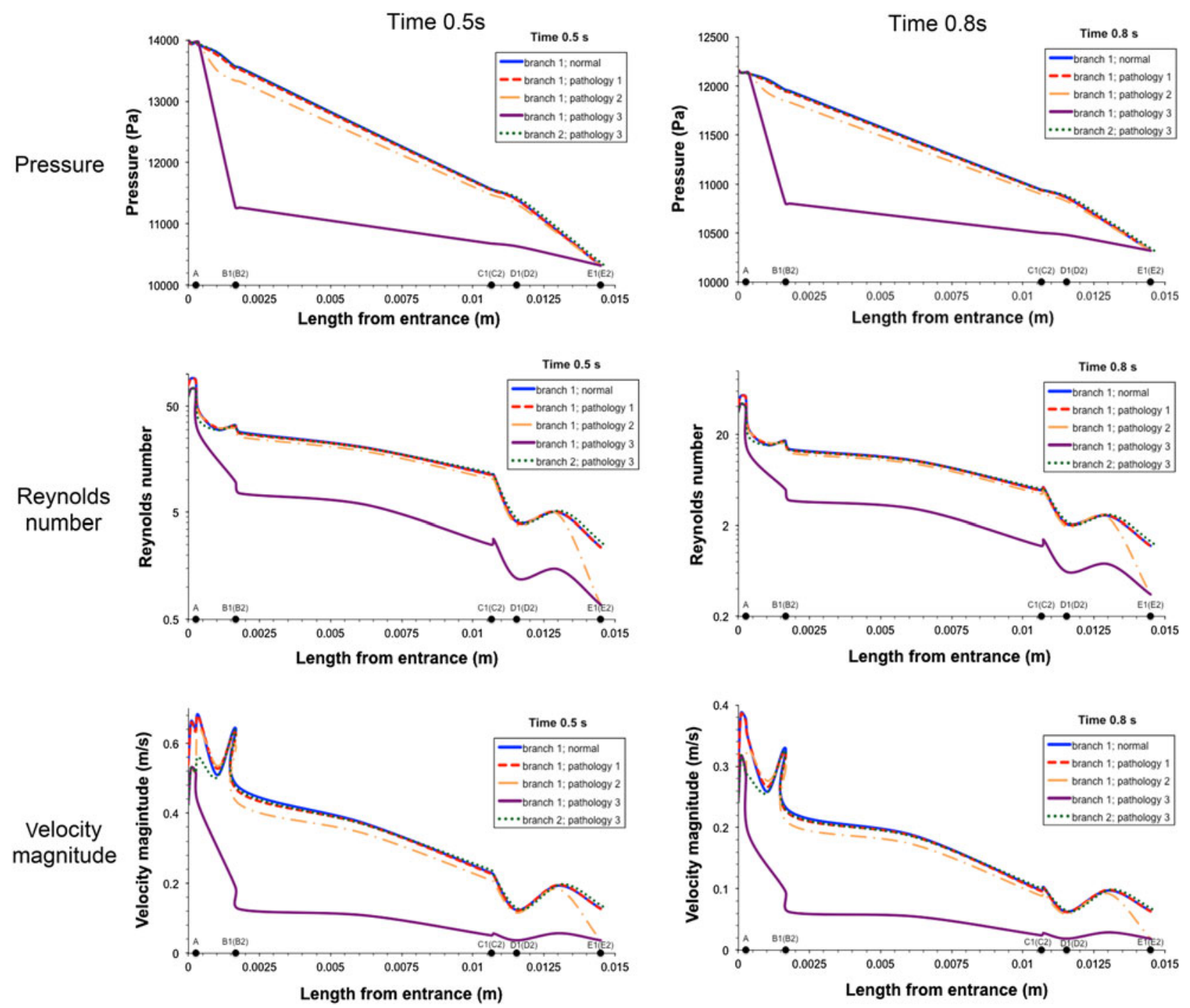

FIGURE 5. Pressure, Reynolds number and velocity magnitude distribution in arterial tree in points defined in Fig. 1 for two different time instances.

intrarenal hemodynamics omitting the regions of high interest involved directly in the pathogenesis.

The model presented here has a significant advantage. It shows the direct and indirect hemodynamic effects of a virtually applied constriction on the zone of interest. In the presented studies, the pathological constrictions not only reduce the value of the flow rate, but also shift the pulse curve characteristics. Furthermore, the vortex formation in such case is different from the normal condition and influences the flow in the entire kidney. Thus, the global approach of modeling the vascular system in an entire organ gives us additional insight into the effects on hemodynamics in not directly affected regions.

All these findings may have some potential diagnostic and clinical implications. In silico simulations of particular or not easily diagnosable conditions, such as stenosis with atypical and complex geometry and localization or modeling of initial pathological stages, could help us to perform quantitative and qualitative flow analysis in well-defined areas and subsequently identify and validate new diagnostic parameters and procedures in the field of sonography and magnetic resonance. Moreover, the analysis of the characteristics of simulated flows of intrarenal and extratrenal stenosis and their correction could enable the analysis of the effects of luminal geometry and surface properties and give rise to the development of optimal stents, endoluminal angioplasty procedures and operative techniques in vascular surgery.

The presented work is a first step toward a challenging project aiming at the creation of a fully mechanistic, accurate, and clinically relevant model of the human renal vasculature fulfilling all the requirements 


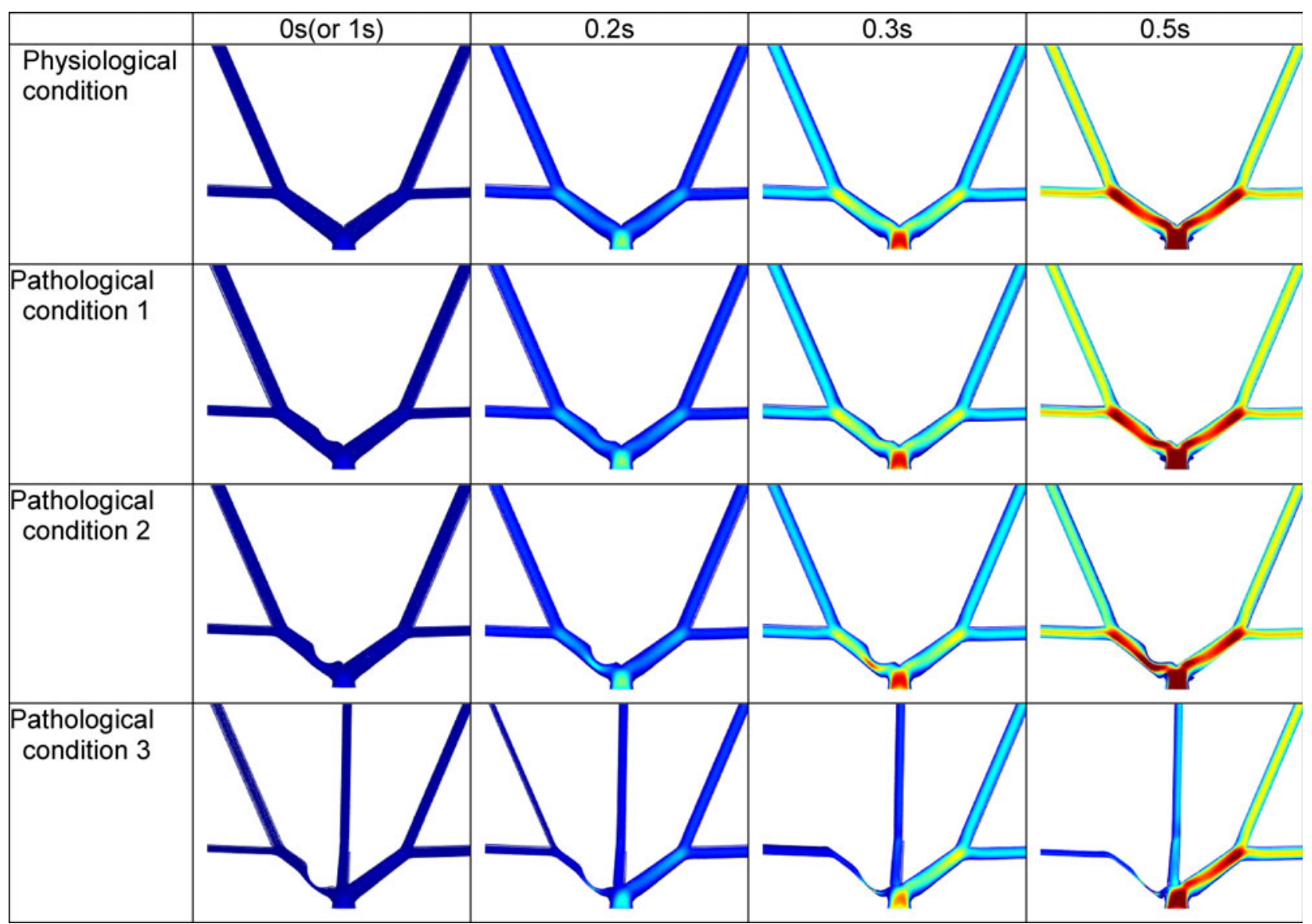

FIGURE 6. Velocity streamlines for physiological and pathological conditions in a middle cut plane.

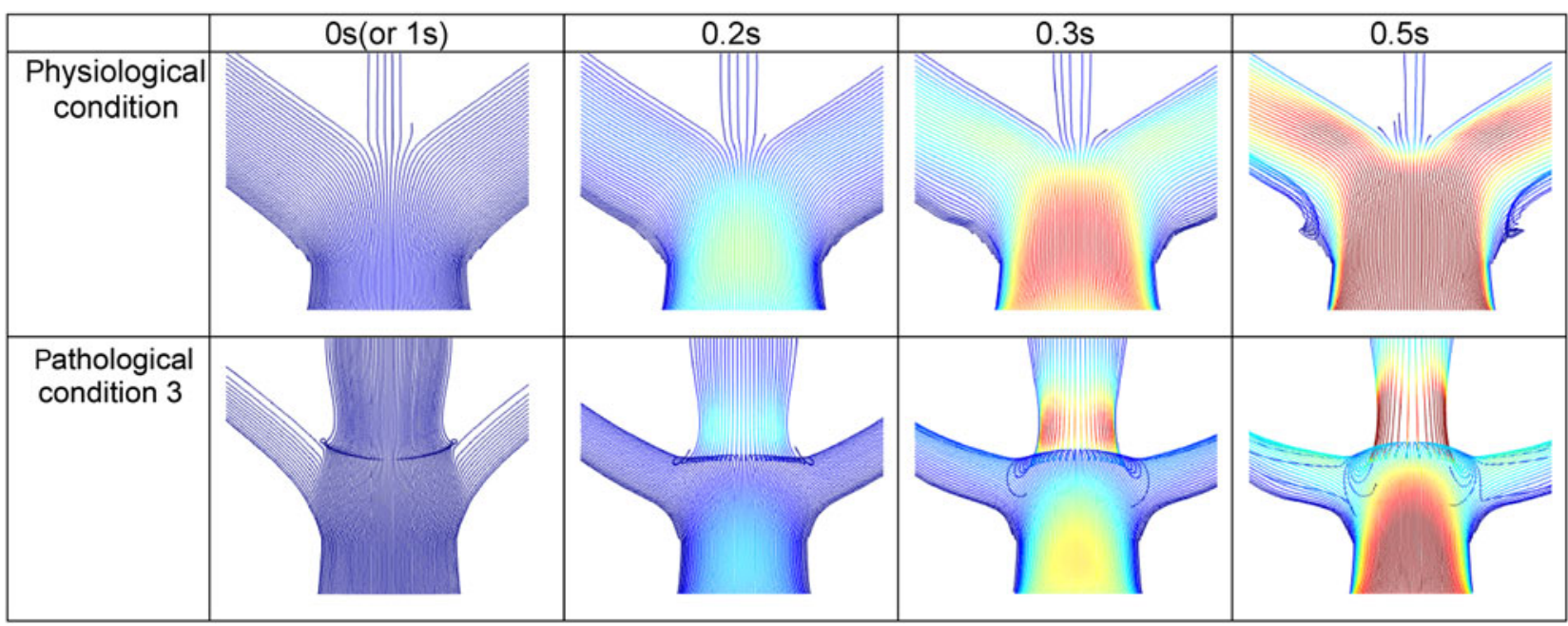

FIGURE 7. Velocity streamlines for physiological and pathological conditions in the off-center plane.

to perform realistic simulations of pathological conditions. To achieve this, many steps still have to be taken. First, the model has to be finalized by solving the 3D configuration problem, and adding the remaining vessels, namely, the interlobular arteries and the afferent and efferent arterioles together with the glomerulus. This way, a complete lobular vascular unit would be created. This unit could be later multiplied by the number of lobules in the human kidney, varying between eight and eighteen according to Dworkin 

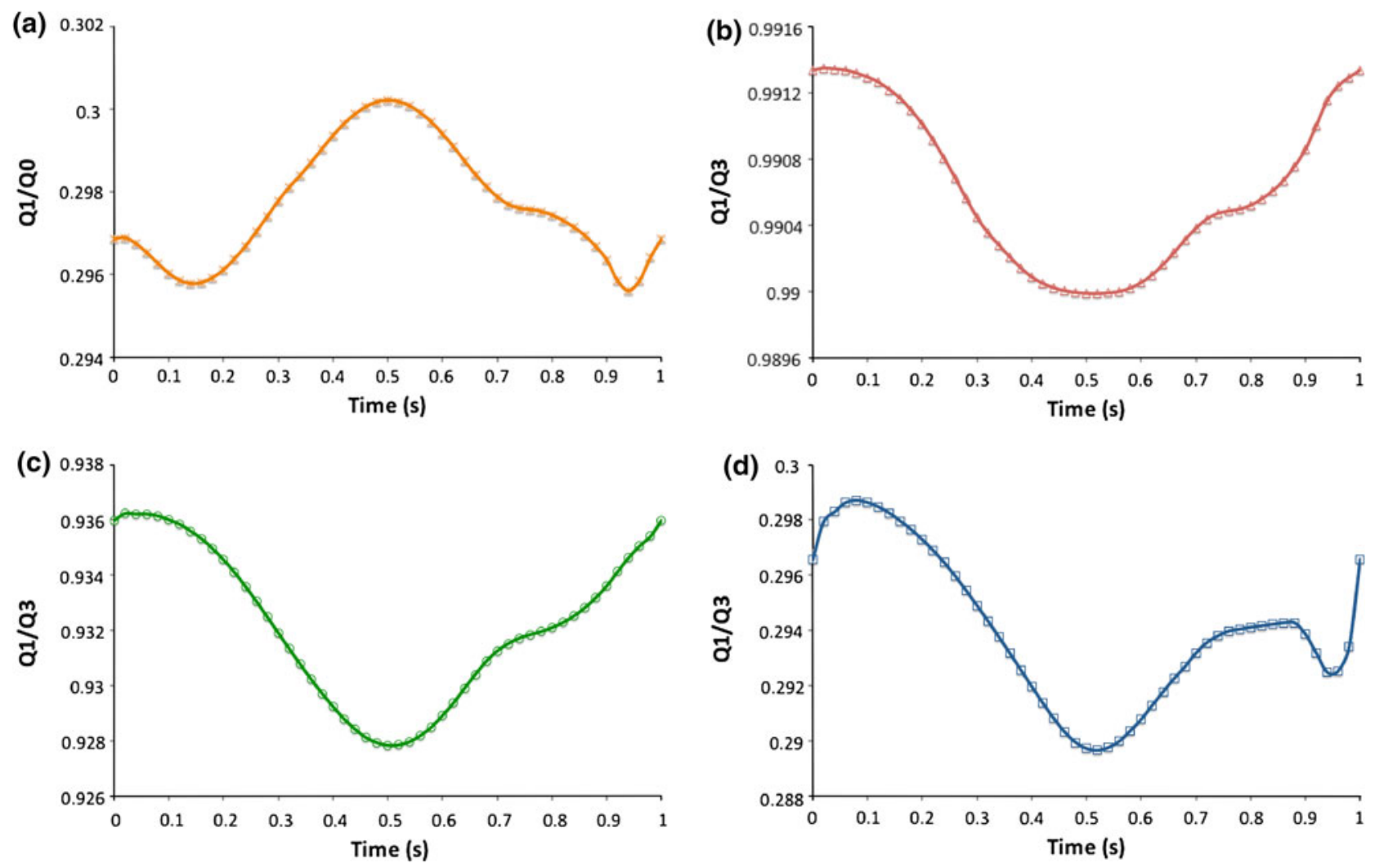

FIGURE 8. Mass flow rate ratio: (a) in branch one and at the entrance, (b-d) at branch one and three; (b) pathological condition 1; (c) pathological condition 2; (d) pathological condition 3. Flow rates at branch one and three are the same at normal conditions.

et al. ${ }^{12}$ Segmental arteries linking these lobules to the main renal artery should also be introduced in order to complete the arterial tree of the human kidney. After that, an elastic component defining the vessel walls and giving them their physiologic compliance would be of interest. Also, blood could be defined in a more complex way, using the immersed body law to predict the flow regime and taking into account regional viscosity dependent variations of the hematocrit. Finally, the obtained simulations should be validated with real data taken from measurements during duplex-sonographic examinations of the kidney. ${ }^{34}$

\section{ELECTRONIC SUPPLEMENTARY MATERIAL}

The online version of this article (doi: 10.1007/s10439-013-0865-8) contains supplementary material, which is available to authorized users.

\section{REFERENCES}

${ }^{1}$ Alpers, C. E. The kidney. In: Robbins and Cotran Pathologic Basis of Disease, 7th ed, edited by V. Kumar, A. K.
Abbas, and N. Fausto. Philadelphia: Elsevier Saunders, pp. 955-1021, 2005.

${ }^{2}$ Asghar, M., K. Ahmed, S. S. Shah, M. K. Siddique, P. Dasgupta, and M. S. Khan. Renal vein thrombosis. Eur. J. Vasc. Endovasc. Surg. 34:217-223, 2007.

${ }^{3}$ Avasthi, P. S., E. R. Greene, and W. F. Voyles. Noninvasive Doppler assessment of human postprandial renal blood flow and cardiac output. Am. J. Physiol. Renal. 252(6):F1167-F1174, 1987.

${ }^{4}$ Bézy-Wendling, J., and M. Kretowski. Physiological modeling of tumor-affected renal circulation. Comput. Methods Prog. Biol. 91:1-12, 2008.

${ }^{5}$ Cai, S., Y.-S. Ouyang, J.-C. Li, Q. Dai, L. Tan, Y. Xia, Z.-H. Xu, H.-J. Li, and Y.-X. Jiang. Evaluation of acute renal thrombosis or embolism with color Doppler sonography. Clin. Imaging 32:367-371, 2008.

${ }^{6}$ Casellas, D., and L. G. Navar. In vitro perfusion of juxtamedullary nephrons in rats. Am. J. Physiol. Renal. 15:F349-F358, 1984.

${ }^{7}$ COMSOL Multiphysics Software, Version 4.1, 2010.

${ }^{8}$ Dal Canton, A., R. Stanziale, A. Corradi, V. E. Andreucci, and L. Migone. Effects of acute ureteral obstruction on glomerular hemodynamics in rat kidney. Kidney Int. 12:403-411, 1977.

${ }^{9}$ De Stefano, V., and I. Martinelli. Abdominal thromboses of splanchnic, renal and ovarian veins. Best Pract. Res. Clin. Haematol. 25(3):253-264, 2012.

${ }^{10}$ Despard, R. A., and J. A. Miller. Separation in oscillating laminar boundary layer flows. J. Fluid. Mech. 47:21-31, 1971. 
${ }^{11}$ Dobbeleir, N., P. Vermeersch, and P. Agostoni. Late renal stent thrombosis. Cardiovasc. Revasc. Med. 11:170-171, 2010.

${ }^{12}$ Dworkin, L. D., and B. M. Brenner. The Renal Circulation. In: Brenner \& Rector's the Kidney, 5th ed, edited by B. M. Brenner. Philadelphia PS: WB Saunders Company, pp. 247-285, 1996.

${ }^{13}$ Evans, R. G., G. A. Eppel, W. P. Anderson, and K. M. Denton. Mechanisms underlying the differential control of blood flow in the renal medulla and cortex. J. Hypertens. 22:1439-1451, 2004.

${ }^{14}$ Gärtner, V., and T. K. Eigentler. Pathogenesis of diabetic macro- and microangiopathy. Clin. Nephrol. 70(1):1-9, 2008.

${ }^{15}$ Greene, E. R., P. S. Avasthi, W. F. Voyles, and R. Seigel. Noninvasive versus invasive Doppler renal blood velocity and flow measurements. IEEE Trans. Biomed. Eng. BME 33(3):302-307, 1986.

${ }^{16}$ Hazirolan, R., M. Öz, B. Türkbey, A. D. Karaosmanoggglu, B. S. Oguz, and M. Canyigit. CT angiography of the renal arteries and veins: normal anatomy and variants. Diagn. Interv. Radiol. 17:67-73, 2011.

${ }^{17}$ Imig, J. D., and R. J. Roman. Nitric oxide modulates vascular tone in preglomerular arterioles. Hypertension 19:770-774, 1992.

${ }^{18}$ Iordache, B. E., and A. Remuzzi. Numerical analysis of blood flow in reconstructed glomerular capillary segments. Microvasc. Res. 49:1-11, 1995.

${ }^{19}$ Jennette, J. C., and R. J. Falk. The pathology of vasculitis involving the kidney. Am. J. Kidney Dis. 24(1):130-141, 1994.

${ }^{20}$ Kagadis, G. C., E. D. Skouras, G. C. Bourantas, C. A. Paraskeva, K. Katsanos, D. Karnabatidis, and G. C. Nikiforidis. Computational representation and hemodynamic characterization of in vivo acquired severe stenotic renal artery geometries using turbulence modeling. Med. Eng. Phys. 30:647-660, 2008.

${ }^{21}$ Kane, J., and M. Sternheim. Physics (2nd ed.). New York: Wiley, 1984.

${ }^{22}$ Kassab, G. S. Scaling laws of vascular trees: of form and function. Am. J. Physiol. Heart Circ. Physiol. 290(2):H894 H903, 2006.

${ }^{23}$ Murray, C. D. The physiological principle of minimum work applied to the angle of branching of arteries. J. Gen. Physiol. 9:835-841, 1926.

${ }^{24}$ Nesbitt, W. S., E. Westein, F. J. Tovar-Lopez, E. Tolouei, A. Mitchell, J. Fu, J. Carberry, A. Fouras, and S. P. Jackson. A shear gradient-dependent platelet aggregation mechanism drives thrombus formation. Nat. Med. 15:665673, 2009.

${ }^{25}$ Nordsletten, D. A., S. Blackett, M. D. Bentley, E. L. Ritman, and N. P. Smith. Structural morphology of renal vasculature. Am. J. Physiol. Heart Circ. Physiol. 291:H296H309, 2006.

${ }^{26}$ Odigi, P. I., and M. Marin-Grez. A method for induction of chronic renal failure in rats. Afr. J. Biomed. Res. 6:1-7, 2003.

${ }^{27}$ Pelaez, L. I., L. A. Juncos, J. M. Stulak, L. O. Lerman, and J. C. Romero. Non-invasive evaluation of bilateral renal regional blood flow and tubular dynamics during acute unilateral ureteral obstruction. Nephrol. Dial. Transplant. 20:83-88, 2005.

${ }^{28}$ Pries, A., D. Neuhaus, and P. Gaehtgens. Blood viscosity in tube flow: dependence on diameter and hematocrit. Am. J. Physiol. 263(32):H1770-H1778, 1992.
${ }^{29}$ Qian, Q., N. A. Saucier, and B. F. King. Acute bilateral renal vein thrombosis. Am. J. Kidney Dis. 54(5):975-978, 2009.

${ }^{30}$ Ribeiro, J. A. S., R. A. Ribeiro, A. G. Caetano, A. O. Rodrigues-Filho, and V. P. S. Fazan. Complex distribution of renal vessels. Braz. J. Morphol. Sci. 24(3):157-159, 2007.

${ }^{31}$ Sabbah, H. N., E. T. Hawkins, and P. D. Stein. Flow separation in the renal arteries. Atheroscler. Thromb. Vasc. Biol. 4:28-33, 1984.

${ }^{32}$ Saldarriaga, B., S. A. Pinto, and L. E. Ballesteros. Morphological expression of the renal artery: a direct anatomical study in a Colombian half-caste population. Int. J. Morphol. 26:31-38, 2008.

${ }^{33}$ Thatipelli, M. R., E. A. Sabater, H. Bjarnason, M. A. McKusick, and S. Misra. CT angiography of renal artery anatomy for evaluating embolic protection devices. J. Vasc. Interv. Radiol. 18:842-846, 2007.

${ }^{34}$ Tranquart, F., L. Mercier, P. Frinking, E. Gaud, and M. Arditi. Perfusion quantification in contrast-enhanced ultrasound (CEUS) - ready for research projects and routine clinical use. Ultraschall. Med. 33(1):S31-S38, 2012.

${ }^{35}$ Trayanova, N. A., T. O'Hara, J. D. Bayer, P. M. Boyle, K. S. McDowell, J. Constantino, H. J. Arevalo, Y. Hu, and F. Vadakkumpadan. Computational cardiology: how computer simulations could be used to develop new therapies and advance existing ones. Europace 14(suppl 5):v82-v89, 2012.

${ }^{36}$ Trueta, J., A. E. Barclay, P. M. Daniel, K. J. Franklin, and M. M. L. Prichard. Studies of the intrarenal vascular pattern. In: Studies of the Renal Vascularisation, 1st ed. Oxford: Blackwell, pp. 39-90, 1947.

${ }^{37}$ Turba, U. C., R. Uflacker, U. Bozlar, and K. D. Hagspiel. Normal renal arterial anatomy assessed by multidetector CT angiography: are there differences between men and women? Clin. Anat. 22:236-242, 2009.

${ }^{38}$ Tylicki, L., B. Rutkowski, and W. H. Hörl. Multifactorial determination of hypertensive nephroangiosclerosis. Kidney Blood Press. Res. 25(6):341-353, 2002.

${ }^{39}$ Ulfendahl, H. R., and M. Wolgast. Renal Circulation and Lymphatics. In: The Kidney: Physiology and Pathophysiology, 2nd ed, edited by D. W. Seldin, and G. Giebisch. New York: Raven Press, pp. 1017-1047, 1992.

${ }^{40}$ Urbieta-Caceres, V. H., R. Lavi, X.-Y. Zhu, J. A. Crane, S. C. Textor, A. Lerman, and L. O. Lerman. Early atherosclerosis aggravates the effect of renal artery stenosis on the swine kidney. Am. J. Physiol. Renal Physiol. 299:F135F140, 2010

${ }^{41}$ US Renal Data System USRDS Annual Data Report, 2011.

${ }^{42}$ Vasava, P., P. Jalali, and M. Dabagh. Pulsatile blood flow simulations in aortic arch: effects of blood pressure and the geometry of arch on wall shear stress. In: IFMBE Proceedings, Vol. 22, edited by S. J. Vander, P. Verdonck, M. Nyssen, and J. Hauseisen. Berlin: Springer, pp. 1926-1929, 2008.

${ }^{43}$ Weld, K. J., S. B. Bahyani, J. Belani, C. D. Ames, G. Hruby, and J. Landman. Extrarenal vascular anatomy of kidney: assessment of variations and their relevance to partial nephrectomy. Urology 66(5):985-989, 2005.

${ }^{44}$ Yamamoto, T., Y. Ogasawara, A. Kimura, H. Tanaka, O. Hiramatsu, K. Tsujioka, M. J. Lever, K. H. Parker, C. J. Jones, C. G. Caro, and F. Kajiya. Blood velocity profiles in the human renal artery by doppler ultrasound and their relationship to atherosclerosis. Atheroscler. Thromb. Vasc. Biol. 16:172-177, 1996. 
${ }^{45}$ Yamamoto, T., Y. Ogasawara, A. Kimura, H. Tanaka, O. Hiramatsu, K. Tsujioka, M. J. Lever, K. H. Parker, C. J. H. Jones, C. G. Caro, and F. Kajiya. Blood velocity profiles in the human renal artery by doppler ultrasound and their relationship to atherosclerosis. Arterioscler. Thromb. Vasc. Biol. 16(1):172-177, 1996.

${ }^{46}$ Yamamoto, T., H. Tanaka, C. J. H. Jones, M. J. Lever, K. H. Parker, A. Kimura, O. Hiramatsu, Y. Ogasawara, K. Tsujioka, C. G. Caro, and F. Kajiya. Blood velocity profiles in the origin of the canine renal artery and their relevance in the localization and development of artherosclerosis. Arterioscler. Thromb. 12(5):626-632, 1992.

${ }^{47}$ Yang, P.-L., D. T. Wong, S.-B. Song, L. Ye, J. Liu, and B. Liu. The feasibility of measuring renal blood flow using transesophageal echocardiography in patients undergoing cardiac surgery. Anesth. Analg. 108(5):14181424, 2009.

${ }^{48}$ Yim, P. J., J. R. Cebral, R. A. Weaver, R. J. Lutz, O. Soto, G. B. C. Vasbinder, V. B. Ho, and P. L. Choyke. Estimation of the differential pressure at renal artery stenoses. Magn. Reson. Med. 51:969-977, 2004.

${ }^{49}$ Zhang, Q.-L., and D. Rothenbacher. Prevalence of chronic kidney disease in population-based studies: systematic review. BMC Public Health 8(117):1-13, 2008.

${ }^{50}$ Zimmerhackl, B., R. Dussel, and M. Steinhauser. Erythrocyte flow and dynamic hematocrit in the renal papilla of the rat. Am. J. Physiol. Renal. 18:F898-F902, 1985. 